\title{
Os impactos dos Royalties do Petróleo em gastos sociais no Brasil: Uma análise usando Propensity Score Matching
}

\author{
Felipe de Sá Tavares* \\ Alexandre Nunes de Almeida **
}

Resumo: O presente trabalho tem como objetivo quantificar e analisar os impactos nos gastos com educação e saúde dos municípios brasileiros que receberam royalties do petróleo e compará-los aos seus pares que não receberam os royalties. Para auferir se houve aumento dos gastos dos indicadores sociais utilizou-se a metodologia do estimador de Diferença nas Diferenças (DID), conjugado com a técnica de Emparelhamento por Escore de Propensão (Propensity Score Matching), ferramenta a qual emparelha as características de municípios de forma a criar um grupo de controle e outro de tratamento, possibilitando a comparação ao reduzir possíveis vieses de seleção. Foi averiguado que os royalties do petróleo aumentam, em média, os gastos com educação e saúde nos municípios beneficiários, em $\mathrm{R} \$ 2$ bilhões e $\mathrm{R} \$ 1.97$ bilhões ao longo do período de 10 anos, respectivamente. No entanto, o impacto dos royalties não se traduziu diretamente no aumento do desenvolvimento social medido pelo IDH.

Palavras-Chaves: Petróleo, Royalties, Emparelhamento.

Classificação JEL: R53, Q48, C15,

\footnotetext{
* Graduado em Ciências Econômicas na Universidade Federal de São Carlos - Campus Sorocaba. E-mail: ftavares29@ gmail.com

** Professor da Escola Superior de Agricultura “Juiz de Queiroz”. Universidade de São Paulo - Campus Piracicaba. E-mail: alex.almeida859@gmail.com
} 


\section{Introdução}

Em 2006, com a descoberta do Pré-Sal na costa brasileira e reservas da ordem de mais 100 bilhões de barris, o Brasil ganhou destaque internacional podendo coloca-lo entre os dez maiores produtores do mundo. Essa importante descoberta, contudo, trouxe uma discussão ampla entre os governos, acadêmicos e sociedade civil sobre como será a lavra do óleo, e principalmente, quem ganhará com os benefícios dessa produção, ou seja, os royalties do petróleo (Queiroz e Postali, 2010).

Os frutos do petróleo são muitos, sendo diretamente, royalties, participações especiais, bônus de assinatura e obrigações sobre a área de retenção. De forma indireta, projetar-se-ia aumento da infraestrutura, desenvolvimento tecnológico, aumento da renda, arrecadação tributária, entre outros (Quintela, 2000). O debate sobre esses dividendos ganham espaço a partir de 1997, ano o qual foi criada a lei $\mathrm{n}^{\circ}$ 9.478, conhecida como Lei do Petróleo. Aumentou-se de $5 \%$ para $10 \%$ a alíquota de cálculo do pagamento dos royalties, fato que teve dois objetivos, primeiro indenizar a população das regiões produtoras devido aos impactos sociais, econômicos e ambientais causados pela atividade de extração e produção (E\&P), e segundo ser uma compensação financeira pela privação que as gerações futuras terão dos recursos do petróleo, almejando uma produção sustentável (Pacheco, 2003).

Como os efeitos gerados pela atividade petrolífera são de grande valor econômico, a discussão sobre o destino dos recursos é protagonizada por dois grupos, um formado pelos estados e municípios produtores, logo beneficiários dos recursos; e aquele constituído por estados e municípios não produtores, e consequentemente os não beneficiários dos recursos, discussão conhecida como "Questão Ibsen" (Freitas, 2009)1.

É notável a evolução do setor petrolífero no Brasil, a qual é acompanhada por um crescimento expressivo dos valores pagos em royalties. Entre 2000 e 2009 , ocorreu um aumento de $1.318 \%$, com projeção de ultrapassar cifras da ordem de $\mathrm{R} \$ 50$ bilhões em 2020 a partir da extração do pré-sal (Nogueira, 2011). Um argumento muito utilizado por municípios não produtores para uma divisão igualitária dos recursos se baseia no aumento de 940\% entre 2000 e 2009 na arrecadação dos municípios beneficiados pelos efeitos indiretos da atividade petrolífera. Embora os dois grupos tenham argumentos para se auto defender com relação aos destinos dos royalties, um fato comum que não é abordado é a gestão desses recursos, ou seja, de que forma os municípios deveriam investir esses dividendos, sendo esta a maior falha da "LP" (Pacheco, 2005). Por exemplo, se são inegáveis as evoluções, tanto dos valores recebidos com royalties e aumentos expressivos dos orçamentos dos municípios produtores de petróleo, estes, como Macaé e Campo de Goytacazes no estado do Rio de Janeiro, tiveram desempenho negativo no desenvolvimento social, perdendo sete e dez posições

\footnotetext{
1 Questão Ibsen, foi o nome como ficou conhecida proposta de lei $\mathrm{n}^{\circ} 12.351$ de 22 de setembro de 2010 , do Deputado Ibsen Pinheiro (PMDB-RS), que tem como objetivo alterar a partilha dos recursos oriundos do petróleo, dando direito de recebimento a todos os estados e municípios brasileiros.

2 Lei do Petróleo, lei n9.478/97.
} 
no ranking do IDH-municipal, respectivamente (Pacheco, 2003).

O presente trabalho tem como objetivo quantificar e analisar os impactos nos gastos com educação e saúde dos municípios brasileiros que receberam royalties do petróleo e compará-los aos seus pares que não receberam os royalties. A hipótese que norteia esse trabalho é que mesmo com as ineficiências encontradas na gestão pública, o recebimento dos royalties, dado pela sua importância e magnitude, pode contribuir significativamente para o aumento dos gastos com saúde e educação nos municípios. Para mensurar esse impacto, utilizou-se a metodologia do estimador da Diferença nas Diferenças (Differences-in-Differences), conjugado com a técnica de Emparelhamento por Escore de Propensão (Propensity Score Matching), ferramenta a qual emparelha as características de municípios de forma a criar um grupo de controle, possibilitando a comparação entre recebedores e não recebedores por eliminar assim possíveis vieses adjacentes de seleção amostral.

Este trabalho está estruturado em cinco seções, além da introdução. A segunda seção abrange a revisão de literatura; e na terceira, a metodologia. Os resultados são apresentados na seção quatro, e na quinta seção, são apresentadas as considerações finais.

\section{Breve Revisão de Literatura}

No Brasil, são ainda escassos os trabalhos acadêmicos sobre os benefícios econômicos do setor petrolífero. Os poucos estudos existentes focam no estado do Rio de Janeiro, já que é o maior produtor de petróleo do país. Em geral, observase que os dividendos adjacentes dos royalties da extração do petróleo têm sido direcionados pelos gestores públicos para sanar dívidas do que diretamente em investimento sociais para a população. Segundo Quintela (2000), os royalties foram imprescindíveis para a saúde financeira do estado do RJ. Ele concluiu que os royalties foram responsáveis por estabilizar o orçamento do estado e consequentemente enquadrando-o na $\mathrm{LRF}^{3}$ (Lei de Responsabilidade Fiscal). Ademais, os royalties contribuíram também para aumento nos investimentos em infraestrutura, saneamento básico e segurança pública. Pacheco (2003), analisando também o impacto dos royalties em municípios do Rio de Janeiro, encontrou que durante o período de 1997 a 2000, os recursos foram direcionados mais no equilíbrio orçamentário estadual e dos municípios beneficiários do que em investimentos em programas sociais, como esperado. Não se encontrou uma resposta positiva nos indicadores sociais entre os municípios analisados.

Mais recentemente, Postali (2007) concluiu que os municípios que são beneficiários dos royalties tiveram desempenho menor que os não beneficiários, indicando a presença regional da "Maldição dos Recursos Naturais". Tal fato é explicado devido à distribuição desproporcional das bacias de petróleo no Brasil, que são concentradas no estado do RJ, tornando a atividade muito importante em

\footnotetext{
3 A Lei de Responsabilidade Fiscal impõe limites ao endividamento das esferas políticas brasileiras de forma que cada unidade de gasto deve, obrigatoriamente, ser justificada por uma unidade de recursos, sem comprometer outros gastos ou receitas. A lei também estabelece o respeito aos mandatos dos gestores para que as contas públicas estejam saudáveis na transição do mandato.
} 
nível regional e não nacional. Postali (2011) também analisou o desenvolvimento social gerado pela atividade petrolífera nos municípios beneficiários por royalties usando o Índice FIRJAN de desenvolvimento Social (IFDM). Ele concluiu que o recebimento dos royalties não teve efeito significativo nos indicadores de saúde e educação. Similarmente, Nogueira (2011) também estudou o impacto gerado pelos royalties nas variáveis de PIB per capita e desigualdade social no ano de 2001 e aferiu que não houve um desenvolvimento social perceptível nos municípios brasileiros beneficiários.

No âmbito internacional, contudo, observa-se outra dimensão quanto ao uso dos royalties. A Arábia Saudita antes do petróleo era um país agrário e préindustrial com uma renda per capita aproximadamente de $\$ 2500,00$. A partir de 1973 com os choques do petróleo que inflacionaram excessivamente os preços dos barris de petróleo a renda do país se multiplicou, podendo ser notado pelo aumento da população e da renda per capita. Em 1973 a população saudita era de aproximadamente 6,8 milhões e em 1995 aproximadamente 18,7 milhões, variando em $275 \%$ para cima; enquanto a renda per capta que em 1973 era de $\$ 2.500,00$ passou para $\$ 9.000,00$ em 1998 , aumentando em $360 \%$. Os recursos gerados também, segundo o autor, foram responsáveis pelo desenvolvimento das áreas de infraestrutura e social (educação e saúde) (Cordesman, 2003).

Segundo Halvor et al (2011), a Noruega também conseguiu se destacar dos outros países com grandes reservas de petróleo devido a superioridade das suas instituições e por já ser uma nação industrial desenvolvida, dessa forma os recursos do petróleo, no caso os royalties, geraram mais desenvolvimento, pois possibilitou incentivar, principalmente, a educação e a saúde. Com esse advento a Noruega não se tornou dependente do recurso, mas sim, desenvolveu uma indústria de ponta para suprir a demanda da atividade petrolífera, sendo, então, a primeira nação a quebrar com a "Maldição dos Recursos Naturais". Os autores concluem que o bom resultado norueguês se dá pela sua economia já ser desenvolvida, independente e, principalmente, por ter baixos níveis de corrupção.

\section{Metodologia}

\subsection{Avaliação de Tratamento}

Em termos gerais, a avaliação de tratamento (ATE) implica na mensuração da diferença dos resultados de interesse entre agentes que participam de um tratamento (uma intervenção a ser avaliada) e outro que não participa. Na literatura ATE, os "não participantes" são denominados como "contra factuais" ou grupo de controle. Formalmente, para um município $i$ em um período $t$, a média condicional do efeito de tratamento (ATE) é dada por:

$$
A T E=E\left[Y_{i}^{T} \mid X, D_{i}=1\right]-E\left[Y_{i}^{C} \mid X, D_{i}=0\right]
$$


onde $\mathrm{Y}_{\mathrm{i}}$ é o resultado em potencial, o impacto a ser medido, $\mathrm{D}_{\mathrm{i}}$ é uma variável binária que indica se o município recebe o tratamento, $\left(D_{i}=1\right)$, e $\left(D_{i}=0\right)$ se não participa do tratamento. $\mathrm{O}$ vetor $\mathrm{X}$ representa um conjunto de covariadas comuns entre os municípios.

Claramente, ambos os resultados não podem ser observados ao mesmo tempo para o mesmo município $i$, constituindo-se como o principal problema analítico em avaliação de impacto (Ravallion, 2008). Usando um resultado de um não participante qualquer como aproximação para o segundo termo em (1), não é recomendado, por ele ou ela possuírem características (e.g., nível de educação, população, grau de urbanização) completamente distintas dos agentes que receberam o tratamento gerando um forte viés de seleção (Angrist e Pischke, 2009). Nota-se que características que geram vieses influenciam apenas elementos observáveis afetando, consequentemente, os impactos da intervenção (Cerdán-Infantes et al., 2009). Duflo et al. (2008) mostra como o viés de seleção pode afetar o resultado do tratamento através da adição e subtração do termo em (1), i.e.:

$$
\begin{aligned}
\mathrm{ATE} & =\mathrm{E}\left[\mathrm{Y}_{\mathrm{i}}^{\mathrm{T}} \mid \mathrm{X}, \mathrm{D}_{\mathrm{i}}=1\right]-\mathrm{E}\left[\mathrm{Y}_{\mathrm{i}}^{\mathrm{C}} \mid \mathrm{X}, \mathrm{D}_{\mathrm{i}}=1\right]-\mathrm{E}\left[\mathrm{Y}_{\mathrm{i}}^{\mathrm{C}} \mid \mathrm{X}, \mathrm{D}_{\mathrm{i}}=0\right]+\mathrm{E}\left[\mathrm{Y}_{\mathrm{i}}^{\mathrm{C}} \mid \mathrm{X}, \mathrm{D}_{\mathrm{i}}=1\right] \\
& =\mathrm{E}\left[\mathrm{Y}_{\mathrm{i}}^{\mathrm{T}}-\mathrm{Y}_{\mathrm{i}}^{\mathrm{C}} \mid \mathrm{X}, \mathrm{D}_{\mathrm{i}}=1\right]+\mathrm{E}\left[\mathrm{Y}_{\mathrm{i}}^{\mathrm{C}} \mid \mathrm{X}, \mathrm{D}_{\mathrm{i}}=1\right]-\mathrm{E}\left[\mathrm{Y}_{\mathrm{i}}^{\mathrm{C}} \mid \mathrm{X}, \mathrm{D}=0\right] .
\end{aligned}
$$

O primeiro termo em (2) é a média do tratamento de efeito no grupo tratado (ATET) e o segundo e terceiro termo correspondem ao viés de seleção, o qual captura potenciais diferenças entre grupo de tratamento e de controle. Consequentemente, a estimação do ATE - usando o modelo análogo em (2) - irá produzir estimativas viesadas do verdadeiro impacto da intervenção.

Um experimento aleatório assume que todos os participantes são elegíveis para receber o benefício de uma intervenção (Duflo et al., 2008). Como resultado, recebendo o tratamento ou não, resultados vão ser potencialmente independentes da intervenção; e consequentemente, o viés da seleção não é mais um problema (Angrist E Pischke, 2009). A randomização da intervenção implica que os últimos dois termos em (2), $E\left[Y_{i}^{\mathrm{C}} \mid \mathrm{D}_{\mathrm{i}}=1\right]-\mathrm{E}\left[\mathrm{Y}_{\mathrm{i}}^{\mathrm{C}} \mid \mathrm{D}=0\right]=0$, e o parâmetro de interesse ATE em (2) é finalmente identificado.

Em termos práticos, experimentos aleatórios em intervenções socioeconômicas são difíceis de serem aplicados por serem muito dispendiosos e de difícil controle; assim, a maioria das avaliações de impactos mais recentes têm sido executadas através de desenho amostrais chamados de "quasi experimental" mensurando os efeitos do tratamento médio sobre o grupo dos tratados (ATET) apenas (Ravallion, 2008). O impacto através do ATET é dado pelo primeiro termo na parte direita em (2) e é estimado como:

$$
A T E T=E\left[Y_{i}^{T}-Y_{i}^{C} \mid X, D_{i}=1\right]=E\left[Y_{i}^{T} \mid X, D=1\right]-E\left[Y_{i}^{C} \mid X, D_{i}=1\right]
$$


Note que novamente ambos os resultados não são observados ao mesmo tempo e, como resultado, a situação contra factual $\mathrm{E}\left[\mathrm{Y}^{\mathrm{C}} \mid \mathrm{X}, \mathrm{D}=1\right]$ precisa ser construída (Caliendo e Kopeinig, 2005). O Propensity Score Matching (PSM) ou Emparelhamento por Escore de Propensão, e a ser discutido adiante, é o método que não somente permite a construção de um grupo contra factual (controle), mas também auxilia eliminar possíveis vieses da seleção amostral nas variáveis observadas (Rosenbaum e Rubim, (1983); Imbens e Wooldridge, (2008)).

\subsection{Emparelhamento por Escore de Propensão}

O principal objetivo do método por Emparelhamento usando Escore de Propensão (Propensity Score Matching) é gerar um "escore" igual ao da probabilidade de recebimento de tratamento, considerando ambos tratados e não tratados a partir de um determinado conjunto de covariadas pré-determinadas (Cameron e Trivedi, 2005) ${ }^{4}$. Em princípio, qualquer modelo binário de escolha pode ser usado para medir a probabilidade de participar de uma intervenção ou não, mas regressões logísticas têm sido mais utilizadas (Becker e Ichino, 2002).

Após a estimativa do modelo de escolha binária incluindo participantes e não participantes e assumindo que as três condições acima são satisfeitas, o próximo passo é construindo o grupo de tratamento com os não tratados usando o escore de propensão (probabilidades esperadas) gerando assim a situação contra factual. Na literatura de avaliação de impacto, muitas técnicas de emparelhamento (matching) estão disponíveis, e não existe uma que se sobressaia sobre a outra (Caliendo e Kopeinig, 2005). O presente trabalho usará as mais frequentemente empregadas como vizinho mais próximo (nearest neighbor ), vizinho mais próximo com distância pré-definida (Radius Matching), regressão não paramétrica (kernel Matching) e regressão local linear $(L L R)^{5}$.

\subsection{Método da Diferença nas Diferenças (DID)}

Uma vez que um grupo de controle é selecionado, o impacto da intervenção na variável de interesse pode ser estimado usando o método Diferença nas Diferenças (DID), assumindo que dados de painel estão disponíveis (Ravaillon, 2008). O uso do DID mensura a diferença entre o resultado dos tratados e do grupo de controle na linha base antes da intervenção versus a diferença entre tratamento e controle após a intervenção (Ravaillon, 2008). O estimador DID é simples e pode ser expresso como:

$$
A T E=E\left[Y_{i}^{T} \mid X, D_{i}=1\right]-E\left[Y_{i}^{C} \mid X, D_{i}=0\right]
$$

\footnotetext{
4 A validade do método PSM deve atender três condições: 1) Independência Condicional; 2) Suporte Comum e; 3) Balanço de Propriedade. As provas formais dessas suposições podem ser encontradas em Rosenbaum e Rubin (1983). 5 Detalhes da implementação de cada metodologia pode ser encontrado em Cameron e Trivedi (2005) e Caliendo e Kopeinig (2005)
} 
A maior vantagem do estimador é controlar vieses de características não observadas e invariantes no tempo (Heckman et al., 1998).

\subsection{Fonte de Dados}

Os dados usados para análise no presente trabalho são dados municipais provenientes de três fontes, a saber, ANP (royalties), IBGE (educação, saúde, PIB, PIB per capta, população) e PNUD (IDH-M) via atlas do desenvolvimento humano. Serão usados os dados referentes aos anos de 2000 e 2009, possibilitando dessa maneira a formação de dados de painéis balanceados dos municípios que possibilitarão a análise de acordo com metodologia proposta.

\section{Resultados}

A tabela 1 abaixo apresenta os resultados obtidos na estimação do modelo Logit utilizado na obtenção dos escores de propensão medindo a probabilidade dos municípios de receber royalties do petróleo para os anos de 2000 e 2009. A diferenciação dos grupos é dada através de um código binário para classificar os municípios que recebiam royalties $(D=1)$ e os que não recebiam $(D=0)$, correspondendo aos grupos de tratamento e de controle, respectivamente. A tabela A1 do apêndice descreve as variáveis utilizadas na estimação do modelo Logit assim como as variáveis de interesse usadas na estimação do impacto dos royalties dos municípios. A tabela A2 do apêndice apresenta as médias e desvio padrão dos dados para os anos de 2000 e 2009. 
Tabela 1 - Resultados da estimação do modelo Logit para o ano de 2000 e 2009.

\begin{tabular}{crrrr}
\hline Variáveis & \multicolumn{2}{c}{ 2000 } & \multicolumn{2}{c}{$\mathbf{2 0 0 9}$} \\
& Coeficientes & $\begin{array}{c}\text { Efeito } \\
\text { Marginal }\end{array}$ & Coeficientes & \multicolumn{1}{c}{$\begin{array}{c}\text { Efeito } \\
\text { Marginal }\end{array}$} \\
\hline autopc & $-7.4804^{*}$ & -0.6844565 & $-2.447618^{*}$ & -0.2919301 \\
& $(1.4435)$ & 0.12823 & $(0.7829427)$ & 0.09382 \\
motospc & -1.6853 & -0.1542092 & $-5.987172^{*}$ & -0.7140969 \\
& $(2.2183)$ & 0.20353 & $(1.310886)$ & 0.15616 \\
onibuspc & $-9.05^{*}$ & -8.276252 & $-102.4997^{*}$ & -12.22525 \\
& $(34.1703)$ & 3.08685 & $(32.28194)$ & 3.81808 \\
caminhoespc & $-32.8907^{*}$ & -3.009476 & $-42.0245^{*}$ & -5.012311 \\
& $(6.5398)$ & 0.59001 & $(8.7672)$ & 1.0168 \\
pibpc & $0.0187^{*}$ & 0.0017179 & $0.011021^{*}$ & 0.0013145 \\
& $(0.0069)$ & 0.00064 & $(0.0032759)$ & 0.00039 \\
obrapc & $-7.7562^{*}$ & -0.7096891 & $-2.25 \mathrm{E}-06$ & -0.0000002690 \\
& $(1.5707)$ & 0.13025 & $(3.14 \mathrm{E}-06)$ & 0.000000 \\
txpopurbana & -0.0703 & -0.0064391 & $1.87708^{*}$ & 0.2238815 \\
\hline N & $(0.1872)$ & 0.01712 & $(0.2109715)$ & 0.02435 \\
\hline LR chi2(7) & & 5564 & & 5564 \\
Pseudo R2 & & 225.68 & & 362.8 \\
Log likelihood & & 0.0543 & & 0.0721 \\
Correct & & -1964.6484 & & -2334.6214 \\
Classified & & $86.57 \%$ & & $83.25 \%$ \\
\hline
\end{tabular}

Nota $1: *, * *, * * *$, significativo aos níveis de $1 \%, 5 \%$ e $10 \%$, respectivamente.

Nota 2: Erros padrão entre parênteses.

Com a estimação do modelo Logit acima foi revelado que para os dados do ano de 2000 todas as variáveis foram significativas a 10\%, exceto motospc e txpopurbana. Enquanto que a estimação com os dados do ano de 2009 apenas obrapc não apresentou-se significativo a $10 \%$, sendo todas as outras variáveis foram significativas ao nível de significância anteriormente citado. De uma forma geral, os resultados não se mostraram sinais esperados. Por exemplo, o sinal negativo da variável autospc indica que quando aumenta o número de automóveis por habitante em uma unidade nos municípios produtores a probabilidade deste receber royalties diminui. Resultados similares também foram encontrados para os outros tipos de veículos incluídos no modelo.

Em relação ao coeficiente da variável PIB per capita (pibpc), este foi único que apresentou sinal positivo nos dois anos de análise, evidenciando que crescimento econômico do município aumenta a probabilidade de receber royalties. Um aumento em uma unidade no PIB municipal de um produtor aumentar a probabilidade de recebimento de royalties em $0,17 \%$ e $0,13 \%$ em 
2000 e 2009, respectivamente. Claramente, é muito difícil prever o sentido causal dessa variável de natureza endógena. Porém, a diminuição da importância do desenvolvimento econômico de um município não produtor na probabilidade deste receber os royalties pode, eventualmente, potencializar a economia do município o qual está inserido.

Como ressaltado, os resultados do modelo Logit não foram esperados, contudo, dentro da literatura de avaliação de tratamento e emparelhamento, o propósito de usar modelos binários discute-se que o critério é apenas para classificação dos grupos e não fazer inferências sobre as estimativas (Caliendo e Kopeinig, 2008). Ademais, para captar com sucesso o efeito do tratamento ao usar dados de experimentos não aleatórios como é o nosso caso, é imperativo garantir que a propriedade de balanço seja satisfeita, isto é, após a realização do pareamento usando as diferentes técnicas propostas, espera-se que as covariadas usadas na estimação não apresentem diferenças estatisticamente significantes depois do emparelhamento (Heinrich et al., 2010).

No presente estudo, amostras pareadas foram construídas a partir dos resultados da regressão logística estimada para o ano de 2009. Os testes da hipótese nula de médias iguais entre tratamento e controle, para cada diferente técnica de emparelhamento utilizada, não foram rejeitados. Assim podemos dizer que conseguimos replicar com sucesso um experimento aleatório, garantindo que municípios tratados e não tratados possuem características idênticas ${ }^{6}$.

$\mathrm{Na}$ tabela 2, tem-se o resultado das cinco técnicas de emparelhamento conjugadas com o estimador diferenças nas diferenças (DID) para os gastos com educação, saúde e IDH. Os resultados de interesse são os diferenciais dos montantes gastos com saúde e educação e o indicador de desenvolvimento humano (IDH) calculado pelo PNUD que varia entre valores de zero e 1.

Em média, os resultados sinalizam que dividendos dos royalties do petróleo aumentariam gastos com saúde e educação da ordem de $\mathrm{R} \$ 2$ bilhões por município durante o período analisado. Note que essas cifras são bem maiores quando comparamos recebedores com não recebedores sem realizar qualquer emparelhamento, valor da ordem de 1,02 bilhão. Ou seja, concluímos que royalties do petróleo contribuem significativamente para aumentar gastos com educação e saúde.

6 Tabelas com os valores dos testes t-student comparando tratamento e controle antes e depois do emparelhamento estão disponíveis para consulta pelo leitor com os autores do artigo. 
Tabela 2 - Impacto (ATET) dos Royalties com gastos em Educação, Saúde e IDH.

\begin{tabular}{rccc}
\hline & $\begin{array}{c}\text { ATETEduc }= \\
\text { Educ2009 }- \text { Educ2000 }\end{array}$ & $\begin{array}{c}\text { ATETSaúde }= \\
\text { Saude2009 }- \\
\text { Saude2000 }\end{array}$ & $\begin{array}{c}\text { ATETIDH }= \\
\text { IDH2009 }- \text { IDH2000 }\end{array}$ \\
\hline Sem emparelhamento & $\approx 1,02$ bilhão & $\approx 1,02$ bilhão & 0.135 \\
$(8,5$ bilhões $)$ & $(8,12$ bilhões $)$ & $(0.0407)$ \\
Vizinho Mais Próximo & $2.121 .300 .000^{*}$ & $1.880 .100 .000^{*}$ & -0.00211 \\
& $(690.088 .612)$ & $(646.457 .905)$ & $(0.00183)$ \\
Caliper (0.029) & $2.202 .900 .000^{*}$ & $2.106 .500 .000^{*}$ & -0.00129 \\
& $(683.745 .473)$ & $(622.748 .193)$ & $(0.00146)$ \\
Kernel $\dagger$ & $2.200 .900 .000^{*}$ & $2.105 .200 .000^{*}$ & -0.001298 \\
& $(683.002 .864)$ & $(622.096 .654)$ & $(0.001467)$ \\
& $2.204 .700 .000^{*}$ & $2.108 .700 .000^{*}$ & -0.001348 \\
& $(683.101 .068)$ & $(622.342 .199)$ & $(0.001481)$ \\
\hline
\end{tabular}

Fonte: Elaborado pelos autores usando o Software STATA 11 (Leuven e Sianesi, 2003).

Nota $1: *{ }^{* *}, * * *$, significativo aos níveis de significância de 1\%, 5\% e 10\%, respectivamente.

Nota 2: Erro padrão entre parênteses.

† O valor usado da largura da janela foi de 0,0149 e foi calculado baseado no método da regra de bolso de Silverman (CAMERON e TRIVEDI, 2005).

Evidentemente, que criticas quanto à destinação desses recursos e mecanismos ótimos de controle da gestão publica referente à qualidade dos serviços prestado a população tem-se tornado pertinentes através de aquecidos debates no congresso nacional. Parece que municípios recebedores dos royalties com essa fonte de renda extraordinariamente significativa, através de seus gestores, não tem sido capazes de prover melhorias nos serviços básicos oferecidos a população de forma a melhorar as condição de desenvolvimento humano como é o caso de Campo dos Goytacazes e Macaé no estado do Rio de Janeiro.

Para captar se municípios recebedores dos royalties tem de fato melhorado as condições de vida, aplicamos o mesmo tratamento metodológico medindo o impacto do IDH nestes como disposto na tabela 2. Nós observamos que os municípios que receberam royalties diminuíram em média o seu IDH em 0.0011 pontos havendo uma piora na qualidade de vida nesses municípios, sinalizando ineficiências e falta de comprometimento dos gestores municipaiscom melhorias na qualidade de vida da população. 


\section{Conclusão}

Essa pesquisa teve como objetivo analisar o impacto dos royalties do petróleo no Brasil. Para a análise e visando um estudo mais robusto foram utilizadas metodologias estatísticas de pareamento conjugadas com o método da diferença nas diferenças. Verificou-se que, ao receber royalties, o município aumentaria significativamente os gastos com educação e saúde. Contudo, o maior entrave ainda consiste na transformação desses gastos em melhoras qualitativas de oferta dos serviços à população, e consequentemente refletindo em melhoria das condições de vida.

A falta de melhoria dos indicadores sociais até então se apoia na corrupção e (des)equilíbrio fiscal por parte dos estados e municípios para justificar o desempenho pífio no desenvolvimento humano. Em relação à corrupção é difícil mensurar o quanto se perde por má gestão, por não existirem registros ou dados oficiais. Em relação ao equilíbrio fiscal, a literatura aponta que gestores municipais em municípios agraciados com os royalties do petróleo estão mais preocupados em sanar dividas de outras fontes do que investir diretamente nos sistemas de saúde e escolas. No caso dos municípios do estado do Rio de Janeiro, por exemplo, a perda da capital federal para Brasília e perda de centro financeiro para São Paulo causaram desequilíbrios fiscais, os quais começaram a ser corrigidos recentemente com os royalties do petróleo, enquadrando-os na "Lei de Responsabilidade Fiscal".

Ademais, essa incapacidade de melhorar a qualidade dos indicadores sociais também pode se apresentar de maneiras distintas, mas com um ponto em comum, isto é, não existem ainda mecanismos eficientes de regulação dos gastos municipais por parte dos entes federais ou estaduais. Os investimentos em educação, por exemplo, são de longo prazo, e para que seja visível a melhora qualitativa dos indicadores educacionais também se faz necessário um acompanhamento periódico desses indicadores nos próximos anos. No caso da saúde, o acompanhamento qualitativo do impacto dos royalties nos municípios é mais difícil já que apresenta características multidimensionais, como por exemplo, politicas de prevenção, saúde da família, reeducação do cidadão a fim de evitar comportamentos de risco, etc.

Em conclusão, a agenda de pesquisa sobre o impacto dos royalties é bastante ampla e deve ser objeto de estudos futuros. A pesquisa possibilitou a conclusão que o Brasil possui um dos maiores motores de desenvolvimento em suas mãos, sendo necessária uma reformulação da Lei do Petróleo no sentido de traçar metas de desenvolvimento via políticas de desenvolvimento social que possam identificar setores estratégicos a serem alvos de maciços investimentos, instituição de um sistema de regulação sério para com os gastos públicos e acoplar os vários setores do país de forma a trazer uma base que possa manter um crescimento sustentável tanto no âmbito econômico quanto no social. 


\section{Referências}

Angrist, J. e Pischke, J. (2009) Mostly Harmless Econometrics, Princeton University Press, Princeton.

Baser, O. Too much ado about Propensity Score Models? Comparing Methods of Propensity Score Matching, (2006) Value in Health. 9(6).

Becker, S. O. e Ichino A., (2002) Estimation of average treatment effects based on propensity scores, The Stata Journal, 2, 358-77.

Caliendo, M. and Kopeining, S. (2005) Some practical guidance for the implementation of propensity score matching. IZA Discussion Paper Series No.1588, Bonn.

Cameron, A. e Trivedi, P. (2005). Microeconometrics: Methods and Applications. Cambridge:Cambridge University Press.

Cerdán-Infantes, P., Maffioli, A. e Ubfal, D. (2008) The impact of agricultural extension services: the sase of grape production in Argentina, Inter-American Development Bank, Office of Evaluation and Oversight OVE Working Papers No. 508, Washington.

Cordesman, Anthony "Saudi Arabia enters the twenty - first century" USA, Greenwood Publishing Group, 2003, 1 edição. Pag. 4 - 24.

Duflo, E, Glennerster, R e Kremer, M, (2008) Using randomization in development economics research: A Toolkit, Ch. 61, in Handbook of Development Economics, (Eds) T. Schultz and J. Strauss, Elsevier, Amsterdam,3895-3962.

Freitas. P.S, de. "Rendas do petróleo, questão federativa e instituição de fundo soberano”. Texto para discussão, Consultoria do Senado Federal, n. 53. 2009. Disponível em: <http://www12.senado.gov.br/publicacoes/estudos-legislativos/ tipos-de-estudos/textos-para-discussao/td-53-rendas-do-petroleo-questaofederativa-e-instituicao-de-fundo-soberano>.

Halvor. M., Karl Ove. M., Ragnar, T. (2011). "Mineral rents and social development in Norway". Memorandum. Departamento de Economia, Universidade de Oslo, $\mathrm{n}^{\circ}$ 14, 2011. Disponível em: <http://www.econstor.eu/handle/10419/47282>. Acesso em: 06 de novembro de 2013.

Heckman, J. J., Ichimura, H., Smith, J. and Todd, P. (1998) Characterizing selection bias using experimental data, Econometrica, 66, 1017-98.

Heinrich, C.; Maffioli, A.; Vásquez, G. (2010). A Primer for Applying Propensity-Score Matching. Technical Notes, No. 161. Washington: Inter-American Development Bank.

Hirano, K., Imbens, G. e Ridder, G. (2000) Efficient estimation of average treatment effect using the estimated propensity score. NBER Technical Working Papers No 0251, National Bureau of Economic Research, Cambridge.

Inbens, G. M. and Wolldridge, J. M. (2008) Recent developments in the econometrics of program evaluation, NBER working papers No. 14251, National Bureau of Economic Research, Cambridge.

Leuven, E.; Sianesi, B. (2003) PSMATCH2: STATA module to perform full 
mahalanobis and propensity score matching, common support graphing, and covariate imbalance testing, Statistical Software Components Series No. S432001, Boston College, Department of Economics, Boston.

Monteiro, N. P. (2010) Using propensity matching estimators to evaluate the impact of privatization on wages, Applied Economics, 42, 1293-1313.

Nogueira. L. C. B., Menezes. T. A. de., "O impacto dos royalties do petróleo e gás natural sobre o PIB per capta, índices de pobreza e desigualdades”. Disponível em: <http://www.bnb.gov.br/content/aplicacao/eventos/forumbnb2011/docs/2011_ os_impactos.pdf>. Acesso em: 27 de Abril de 2012.

Pacheco, C.A.G. - "A Aplicação e o Impacto dos Royalties do Petróleo no Desenvolvimento Econômico dos Municípios Confrontantes Da Bacia De Campos”. Tese de dissertação de monografia de bacharelado em economia do instituto de economia da UFRJ, 2003.

Pacheco, C.A.G. - "O impacto dos royalties do petróleo no desenvolvimento econômico dos municípios da região norte fluminense". Artigo cientifico apresentado no $3^{\circ}$ Congresso Brasileiro de P\&D em Petróleo e Gás, 2005.

Postali. F. A. S., "Efeito da distribuição dos royalties do petróleo sobre o crescimento dos municípios do Brasil". In: Encontro Nacional de Economia, XXXV, 2007, Recife/PE, Anais. Disponível em: <http://econpapers.repec.org/paper/ anpen2007/072.htm>. Acesso em 17 de outubro de 2013.

Postali. F. A. S., Nishijima. M., "Distribuição das rendas do petróleo e indicadores de desenvolvimento municipal no Brasil nos anos 2000's". Estudos Econômicos, São Paulo, V. 41, n 2, Abr - Jun. 2011. Disponível em: <http://www.scielo.br/scielo. php?pid=S0101-41612011000200010\&script=sci_abstract\&tlng=pt $>$. Acesso em: 19 de setembro de 2013.

Queiroz. C. R. A., Postali. F. A. S. “ Roaylties e arrecadação municipal: Apontando Ineficiências do Sistema de Divisão das Rendas do Petróleo no Brasil". Temas de economia aplicada, São Paulo, 359, agosto de 2010. Disponível em:< http://www. fipe.org.br/publicacoes/downloads/bif/2010/8_bif359.pdf>. Acessado em: 22 de novembro de 2013.

Quintela, S.F. - "Os royalties do petróleo e a economia do estado do Rio de Janeiro". TCE - RJ, 2000.

Ravallion, M. (2008) Evaluating anti-poverty programs, chap. 59, in Handbook of Development Economics, (Eds) T. Schultz. and J. Strauss, Elsevier, Amsterdam, pp. 3787-3846.

Rosenbaum, P. and Rubin, D. (1983) The central role of the propensity score in observational Studies for Causal Effects, Biometrika, 70, 41-55.

Tabelas com valores dos Royalties do Petróleo e do Gás Natural, 1999 a 2012. Disponível em ANP, <http://www.anp.gov.br/?pg=59736\&m=royalties\&t1=\&t2=r oyalties\&t $3=\& \mathrm{t} 4=\& a r=0 \& p s=1 \&$ cachebust $=1334544039091>$.

Tabelas com valores de IDH-M, 1991, 2000 e 2010. Disponível em IBGE Cidades, $<$ http://www.ibge.gov.br/cidadesat/xtras/temas.php?codmun=130002\&idtema= 118\&search=amazonas | alvaraes | \%C3\%8Dndice-de-desenvolvimento-humanomunicipal-idhm->. 


\section{Apêndice}

\section{Tabela A1 - Descrição das variáveis}

\begin{tabular}{ll}
\hline \multicolumn{1}{c}{ Variáveis } & \multicolumn{1}{c}{ Descrição } \\
\hline Binario & 1 se o municipio recebe royalties \\
Autopc & número de automóveis por habitante \\
motospc & número de motocicletas por habitante \\
onibuspc & número de onibus por habitante \\
caminhoespc & número de caminhões por habitante \\
Pibpc & pib per capta \\
Obrapc & gasto com obras e instalações por habitante \\
txpopurbana & porcentagem da população que reside na cidade do município \\
Saude & Montante gasto em saúde \\
Educaçao & Montante gasto em educação \\
IDH & IDH municipal \\
\hline
\end{tabular}

Fonte: ANP, IBGE cidades e PNUD.

Tabela A2 - Médias e Desvios Padrões

\begin{tabular}{lcccc}
\hline \multicolumn{1}{c}{ Variáveis } & \multicolumn{2}{c}{$\mathbf{2 0 0 0}$} & \multicolumn{2}{c}{ 2009 } \\
\hline Média & Desvio Padrão & média & desvio padrão \\
\hline motospc & 0,034 & 0,0549 & 0,1197 & 0,1032 \\
onibuspc & 0,026 & 0,0302 & 0,0650 & 0,0384 \\
caminhoespc & 0,0018 & 0,0082 & 0,0020 & 0,00651 \\
pibpc & 0,0097 & 0,01424 & 0,0121 & 0,01316 \\
obrapc & $4,751,42$ & $1,680,80$ & $11,038,18$ & $1,155,331$ \\
txpopurbana & 0,037312 & 0,3667 & $9,446,56$ & 13537,56 \\
Educacao & 0,585418 & 0,2356 & 0,6382 & 0,2204 \\
Saude & $4,234,26$ & $25,800,000$ & $1,130,000,000$ & $8,580,000,000$ \\
IDH & $3,321,62$ & $25,400,000$ & $1,020,000,000$ & $8,160,000,000$ \\
\hline
\end{tabular}

Fonte: ANP, IBGE cidades e PNUD. 